\title{
Characterization of the DGAT1 gene in the Indian buffalo (Bubalus bubalis)
}

\author{
Bina Mishra, Madhu S. Tantia, S.T. Bharani Kumar and Ramesh K. Vijh \\ National Bureau of Animal Genetic Resources, Karnal, Haryana, India.
}

\begin{abstract}
The positional candidate diacylglycerol $O$-acyltransferase (DGAT1) gene affecting milk fat percentage is reported in Indian buffaloes (Bubalus bubalis). A comparison with Chinese buffalo (Bubalus bubalis) revealed eight exonic single nucleotide polymorphisms (SNPs), five of which were non-synonymous. A total of 19 SNPs were observed among diverse buffalo breeds in India. A Unique 22 base insertion has been reported in the intron between exon ten and eleven.
\end{abstract}

Key words: Bubalus bubalis, DGAT1 gene, diacylglycerol $O$-acyltransferase, Indian water buffalo, single nucleotide polymorphisms (SNPs).

Received: November 28, 2006; Accepted: May 14, 2007.

Diacylglycerol $O$-acyltransferase (DGAT1; EC 2.3.1.20) is a microsomal enzyme catalyzing the addition of fatty acyl Co A to 1, 2, diacylglycerol to yield CoA plus triglycerol and is important in lipogenesis in many tissues, including mammary gland (Cases et al. 1998). The DGAT1 gene is a positional candidate gene for milk fat percentage with K232A substitution associated with higher fat percentage in Bos taurus (Winter et al. 2002; Grisart et al. 2004; Kuhn et al. 2004). Kaupe et al. 2004 reported the frequency of this substitution in various cattle breeds and grouped them from very low frequency to fixation in Bos indicus cattle breeds.

There are 10 recognized breeds of water buffalo (Bubalus bubalis), which are adapted to different climates and show large variation in size and have milk productivity ranging from $600 \mathrm{~kg}$ to $2500 \mathrm{~kg}$ per lactation with 6 to 8 percent fat. The Indian water buffalo (Bubalus bubalis) is an important dairy animal in the Indian subcontinent, with buffaloes producing more than $50 \%$ of the total milk in India. In the last two decades the buffalo population in India has registered an annual growth of $1.93 \%$, reaching 97 million in 2003 (Annual Report, 2006) and continuing to be the mainstay of the Indian dairy industry and replacing cattle in several milk producing areas.

The DGATl gene has been shown to be important for milk production, with Smith et al. (2000) having demonstrated defective lactation in DGAT1 -/- female mice. The sequence of the Chinese water buffalo (Bubalus bubalis) DGAT1 gene has recently been reported (NCBI GenBank

Send correspondence to M.S. Tantia. National Bureau of Animal Genetic Resources, Karnal 132001, India. E-mail: mstantia@ yahoo.com.
AY999090). Since buffaloes yield higher milk fat than Bos indicus or Bos taurus cattle we felt that the characterization of DGAT1 in Indian buffalo was necessary. In the research reported in this paper the Indian water buffalo DGAT1 gene sequence was generated and compared with the AY999090 from Chinese buffalo and Bos taurus cattle to record variations.

The DGAT1 gene was characterized in a panel of 24 animals drawn from 6 diverse Indian water buffalo breeds (Murrah, Bhadawari, Tarai, Pandharpuri, Marathwada and Mehsana). The Murrah breed is a Northern Indian large dairy breed while the Bhadawari and Tarai are small breeds adapted to extensive production systems. The Pandharpuri and Marathwada breeds are medium sized buffaloes from central India and the Mehsana breed is a western Indian dairy breed. The DNA was isolated from blood samples and diluted to optimum concentration. The genomic region corresponding to the putative buffalo DGATI gene was amplified by the polymerase chain reaction (PCR) with 16 primer pairs (Table 1) designed for the Bos taurus GenBank AJ318490 DNA sequence. The PCR reactions were performed in $25 \mu \mathrm{L}$ reaction mixtures containing $50 \mathrm{ng}$ of genomic DNA, 4 pmol of each primer, $1.5 \mathrm{mM} \mathrm{MgCl}_{2}, 0.2 \mu \mathrm{M}$ of each dNTP and 1 unit of Taq DNA polymerase (Sigma) and in a model 9700 GeneAmp Cycler (Applied Biosystems, USA) using an amplification protocol consisting of 5 min denaturation at $94^{\circ} \mathrm{C}$ followed by 30 amplification cycles of $94^{\circ} \mathrm{C}$ for $45 \mathrm{~s}, 57^{\circ} \mathrm{C}$ to $55^{\circ} \mathrm{C}$ for $45 \mathrm{~s}$ and $72{ }^{\circ} \mathrm{C}$ for $45 \mathrm{~s}$, with a final extension at $72{ }^{\circ} \mathrm{C}$ for $10 \mathrm{~min}$. The PCR products were separated on $2 \%$ $(\mathrm{w} / \mathrm{v})$ agarose gel (Promega, USA) for examination of specific products of expected sizes. The PCR products were purified using a Montage column (Millipore) and subjected to 
cycle sequencing reactions in a $10 \mu \mathrm{L}$ reaction volume using the BigDye system (Applied Biosystems, USA) following the manufacturer's protocol. Sequencing was carried out using forward and reverse primers (Table 1) on a 3100 Avant automated sequencer (Applied Biosystems, USA). In addition 8 primers (Table 2) were designed for primer walking and used to sequence the larger PCR products. The sequences obtained for each fragment were aligned to obtain a $D G A T 1$ consensus sequence using the $B$. taurus sequence as reference and the SeqScape program v.2 (Applied Biosystems, USA). The single nucleotide polymorphisms (SNPs) detected in the Indian water buffalo DGAT1 gene were further confirmed in additional samples from the panel of 24 Indian water buffalo and the frequencies of the SNPs estimated.

The sequence of 8717 bases for the Indian water buffalo DGAT1 gene has been submitted to the NCBI GenBank with accession number DQ886485. We found that the gene in buffalo coded for 489 amino acids which is similar to Chinese buffalo, cattle and pig. Comparison of the Chinese and Indian water Buffalo sequences revealed eight changes in exonic regions, five of which were non-synonymous at amino acid numbers 98, 154, 367, 370 and 407 (Figure 1). Comparison of the Indian water buffalo DGAT1 sequence with the $B$. taurus sequence revealed 10 synonymous changes in the coding sequence CDS and two nonsynonymous changes. The first non-conservative change was a double SNP at nt6962 and 6963 (adenine/adenine to guanine/cytosine) resulting in the previously reported change of K232A in B. taurus which has been associated with increased fat yield and reduced milk yield (Spelman et al. 2002; Thaller et al. 2003; Weller et al. 2003). All the buf-
Table 2 - Additional primers designed for Primer walking.

\begin{tabular}{llcl}
\hline Serial number & 5' - 3' sequence & Position & Type \\
\hline 1 & gTCCTTCCTgTgggCTATgA & $657-676$ & Forward \\
2 & TCTggAgCTgATCCCAAAAC & $1145-1164$ & Reverse \\
3 & gAAgCTgCTCCTgTCCTgTT & $1601-1620$ & Forward \\
4 & CAggCACAgCCTTCTAgTCC & $2089-2108$ & Forward \\
5 & gTCAgggAgAgggTgACAgA & $2428-2447$ & Forward \\
6 & CTgTTCCAAATTgCCCTCAT & $4425-4444$ & Forward \\
7 & gAACAgACAggCTgCTgAgg & $4960-4979$ & Reverse \\
8 & gAgCTgACTCTgCgCTTTTT & $7384-7403$ & Forward \\
\hline
\end{tabular}

falo breeds (Indian and Chinese) as well as Bos indicus cattle (Kaupe et al. 2004; Lacorte et al. 2006; Tantia et al. 2006) have a fixed $\mathrm{K}$ allele, which may be responsible for higher milk fat in buffalo and $B$. indicus cattle.

Rincón et al. 2006 found that Uruguayan Creole cattle (B taurus) had DGAT1 allele frequencies (A allele $=0.89 ; \mathrm{K}$ allele $=0.11$ ) similar to $B$ taurus beef breeds. The $B$. taurus breeds selected for milk production show more variation in DGAT1 with allele frequencies of some breeds being close to 0.50 for each allele (e.g. German Holstein, A allele $=0.58 ; \mathrm{K}$ allele $=0.42$ ) or higher for the $\mathrm{K}$ allele (e.g. New Zealand Holstein-Friesian, A allele $=0.40 ; \mathrm{K}$ allele $=0.60$; and Jersey, A allele $=0.31 ; \mathrm{K}$ allele $=0.69$ ), which is most likely the result of artificial selection for increasing milk yield and/or high milk fat content (Spelman et al. 2002; Kaupe et al. 2004).

The second amino acid change (R431G) reported by us was the result of two transversions at 8168 and 8170 $(\mathrm{CGC} \rightarrow \mathrm{GGA})$. Grisart et al. 2002 a reported CGC

Table 1 - Primer pairs for amplification of the Indian buffalo (Bubalus bubalis) DGAT1 gene.

\begin{tabular}{|c|c|c|c|c|c|}
\hline Serial number & $5^{\prime} \rightarrow 3^{\prime}$ forward primer & $5^{\prime} \rightarrow 3^{\prime}$ reverse primer & Position (from/to) & Size & Region \\
\hline 1 & ACggCAgTggCgTAgTAgAg & ACgTCTCCgTCCTTgTCTgT & 1 to 286 & 285 & 5' upsteam-Exon 1 \\
\hline 2 & AgAggAggTgCgggATgT & AAATCCAgTTCCCCAggAgT & 218 to 1500 & 1282 & Exon 1-Intron 1 \\
\hline 3 & ggCACACAgTTCACCTgAgA & ggTggCACСТCATTCAACCT & 1295 to 2337 & 1042 & Intron 1 \\
\hline 4 & ACTCCTggggAACTggATTT & CAATCACCATCTgCATgTCC & 1481 to 2893 & 1412 & Intron 1 \\
\hline 5 & TTggCAggTTgTAgCATgAg & gCAAggCCTCCAgTTTTgTA & 2891 to 3394 & 503 & Intron 1 \\
\hline 6 & ggССТСТССССТТАСААААС & CACACACCAATTCAggATgC & 3363 to 4024 & 661 & Intron 1/Exon 2 - Exon 2 \\
\hline 7 & CgTCTCCACTCTCCAggTgT & AgCAgCAgCAAAggACAgAT & 3930 to 5463 & 1533 & Intron 1 - Intron 2 \\
\hline 8 & TggCATCCTgAATTggTgTg & gAAATAACCgTgCgTTgCTT & 4003 to 6003 & 2000 & Exon 2 - Exon 3 \\
\hline 9 & gCAgCAggTTTCTTCTgTCA & gTgAgggCACTgCTTACCAC & 5730 to 6346 & 616 & Intron 2 - Exon/Intron 5 \\
\hline 10 & AAgCAACgCACggTTATTTC & TTCAggAACAgAgACACCACCT & 5984 to 6146 & 162 & Exon 3 - Exon 4 \\
\hline 12 & CATCCTCTTCCTCAAgCTgTTC & TgCCAgAAgTAggTgATggACT & 6765 to 7872 & 1107 & Exon 7 - Exon 14 \\
\hline 13 & CCCTgTgCTACgAgCTCAAC & ggTgATAgACTCggAgTTCCTg & 7120 to 7861 & 741 & Exon 9 - Exon 14 \\
\hline 14 & TgAgCTCATgCAgTTTggAg & CACCAggTACTgAggggAgA & 7710 to 8155 & 445 & Exon 13 - Exon 16 \\
\hline 15 & CTCCCCTCAgTACCTggTgA & CCCgATgATgAgTgACAgC & 8137 to 8370 & 233 & Intron 15/Exon 16- Exon 17 \\
\hline 16 & gATAgTgggCCgCTTCTTC & TgCACAgCACTTTATTgACACA & 8304 to 8717 & 413 & Exon $17-3$ ' downstream \\
\hline
\end{tabular}


Indi an Buf $f$ al o Chi nese Buffalo Bos taurus Sus scrof a Homo sapi ens

I ndi an Buffalo Chi nese Buffalo Bos taurus Sus scrof a Homo sapi ens

I ndi an Buffalo Chi nese Buffalo Bos taurus Sus scrof a Homo sapi ens

Indi an Buffalo Chi nese Buffal o Bos taurus Sus scrof a Homo sapi ens

I ndi an Buffal o Chi nese Buffalo Bos taurus Sus scrof a Homo sapi ens

I ndi an Buffalo Chi nese Buffalo Bos taurus Sus scrof a Homo sapi ens

I ndi an Buffalo Chi nese Buffalo Bos taurus Sus scrof a Homo sapi ens

Indi an Buffalo Chi nese Buffalo Bos taurus Sus scrof a Homo sapi ens

I ndi an Buffalo Chi nese Buffalo Bos taurus

Sus scrof a Homo sapi ens

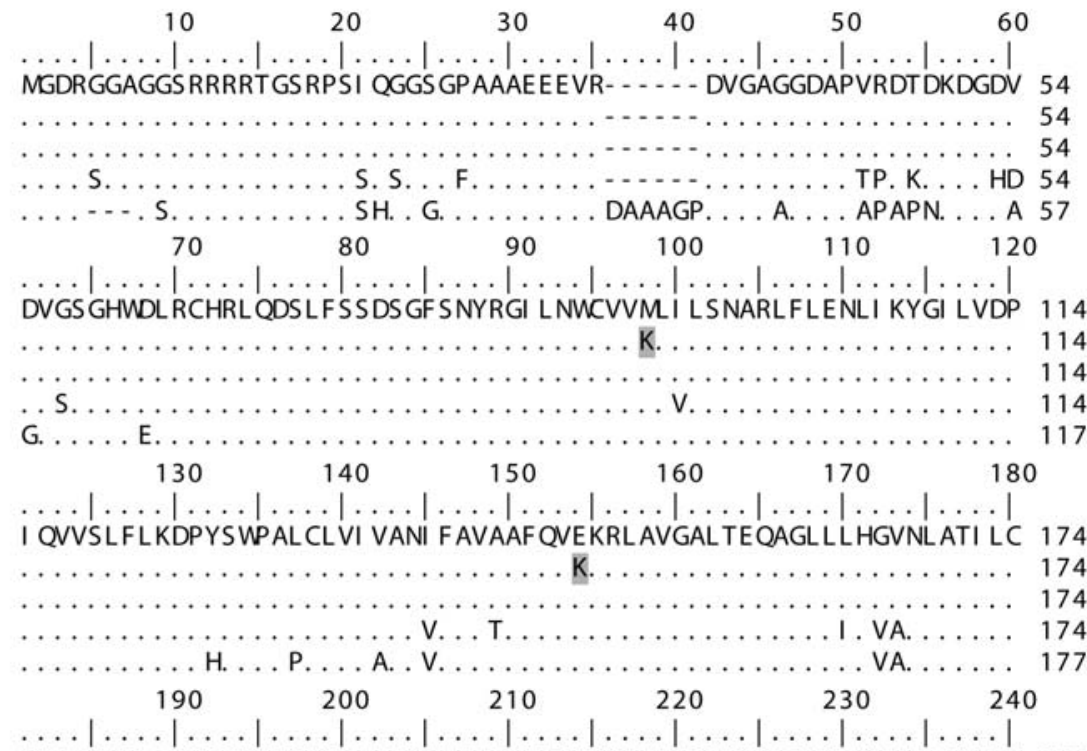

FPAAVAFLLESI TPVGSVLAL WYTI LFLKLFSYRDVNL WCRERRAGAKAKAALAGKKAN 234

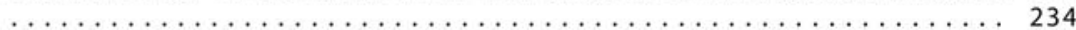

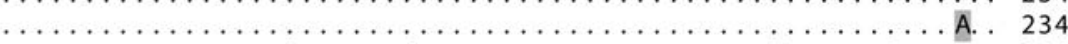
$\ldots \ldots \ldots \ldots \ldots$ L. . . A. . . . . . . . . . . . . . 234

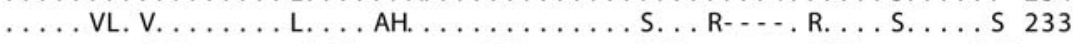
$\begin{array}{llllll}250 & 260 & 270 & 280 & 290 & 300\end{array}$

$\ldots|\ldots| \ldots|\ldots| \ldots|\ldots| \ldots|\ldots| \ldots|\ldots| \ldots|\ldots| \ldots|\ldots|$

GGAAQRTVSYPDNLTYRDL YYFLFAPTLCYELNFPRSPRI RKRFLLRRLLEM FLTQLQV 294

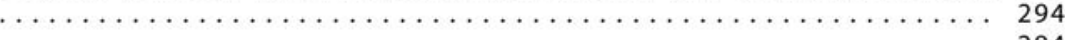

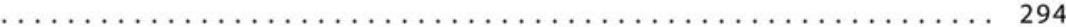

$\ldots \ldots$ HS. . . . . . . L. . . . . S. F. . . . . . . . . . . 294

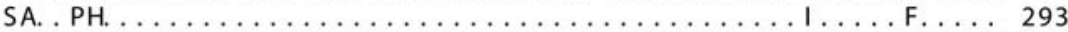
$\begin{array}{llllll}310 & 320 & 330 & 340 & 350 & 360\end{array}$

$\ldots|\ldots| \ldots|\ldots| \ldots|\ldots| \ldots|\ldots| \ldots|\ldots| \ldots|\ldots| \ldots \mid$

GLI QQWMNPAI QNSMKPFKDMDYSRI VERLLKLAVPNHLI WLI FFYWLFHSCLNAVAELM 354

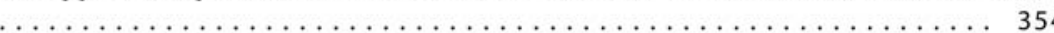

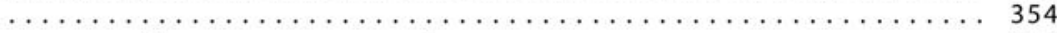

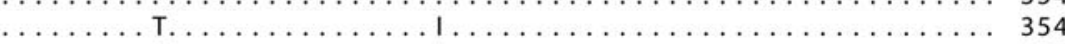

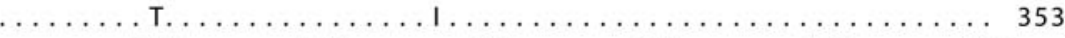

$\ldots|\ldots| \ldots|\ldots| \ldots|\ldots| \ldots|\ldots| \ldots|\ldots| \ldots|\ldots| \ldots|\ldots| \ldots|\ldots| \ldots \mid$

QF GDREFYRDWWNSESI TYFWONWNI PVHKWCI RHFYKPMLRRSSKWAARTAVFLASAF 414

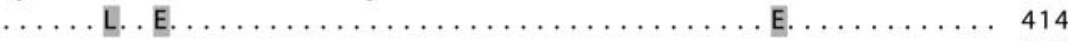

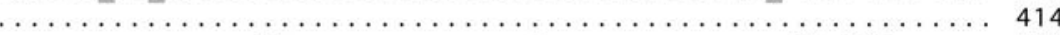

$\ldots \ldots \ldots \ldots \ldots$ V. . . . . . . . . . . . 414 $\ldots \ldots \ldots \ldots \ldots$ V. . . . . . . . . . . . . . . 413 $\begin{array}{llllll}430 & 440 & 450 & 460 & 470 & 480\end{array}$

$\ldots|\ldots| \ldots|\ldots| \ldots|\ldots| \ldots|\ldots| \ldots|\ldots| \ldots|\ldots| \ldots \mid$

FHEYLVSI PLRMFRL WAFTGMMAQ PLAW VGRFFRGNYGNAAVWLSLI I GQPVAVLMYV 474

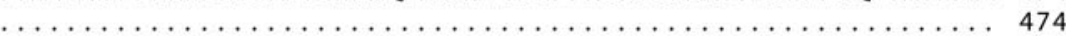

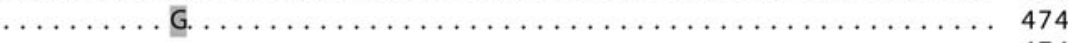

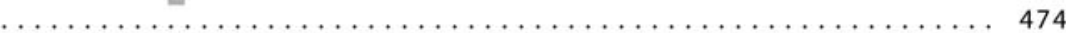

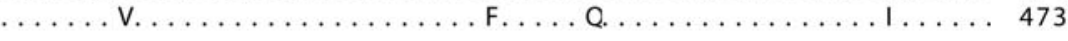

490

$\ldots|\ldots| \ldots \mid$

HDYYVLNREAPAAGT 489

.......... 489

$\ldots \ldots \ldots \ldots, 489$

..... HH. . . T. . A 489

$\ldots \ldots$ Y.... EA 488

Figure 1 - Clustal-W alignment of the DGAT1 amino acid sequence.

(AY065621) change while Winter et al. (2002) reported a GGA (AJ318490) change in B. taurus breeds, whereas Indian and Chinese buffalo had a CGC change coding for Arginine
We found that the Indian buffalo DGAT1 gene sequence was quite close to the Chinese buffalo sequence, with a striking insertion of 22 bases in intron 10 which was unique to Indian buffalo and was also not present in $B$ 
Table 3 - The DGAT1 polymorphism in Indian buffalo (Bubalus bubalis) breeds. Data shown for introns and the 3' untranslated region (UTR).

\begin{tabular}{|c|c|c|c|c|c|}
\hline Region and nucleotide & Position (from/to) & Frequency & Region and nucleotide & Position (from/to) & Frequency \\
\hline Intron 1 & & & Intron 2 & & \\
\hline $1179 *$ & A to $T$ & 0.12 & 5545 & $\mathrm{~T}$ to $\mathrm{C}$ & 0.20 \\
\hline 1195 & $\mathrm{C}$ to $\mathrm{T}$ & 0.11 & & & \\
\hline 1606 & $\mathrm{~T}$ to $\mathrm{C}$ & 0.06 & Intron 3 & & \\
\hline 1784 & $\mathrm{G}$ to $\mathrm{A}$ & 0.07 & 6067 & $\mathrm{C}$ to $\mathrm{T}$ & 0.06 \\
\hline 1875 & $\mathrm{G}$ to $\mathrm{A}$ & 0.13 & & & \\
\hline $2141^{*}$ & $\mathrm{G}$ to $\mathrm{T}$ & 0.04 & Intron 15 & & \\
\hline 2217 & $\mathrm{C}$ to $\mathrm{T}$ & 0.14 & 8087 & $\mathrm{C}$ to $\mathrm{T}$ & 0.08 \\
\hline 2394 & $\mathrm{C}$ to $\mathrm{T}$ & 0.08 & & & \\
\hline 3057 & A to $\mathrm{G}$ & 0.21 & Intron 16 & & \\
\hline $3096^{*}$ & $\mathrm{G}$ to $\mathrm{T}$ & 0.18 & 8259 & $\mathrm{G}$ to $\mathrm{A}$ & 0.41 \\
\hline 3627 & $\mathrm{C}$ to $\mathrm{T}$ & 0.20 & & & \\
\hline 3674 & $\mathrm{G}$ to $\mathrm{A}$ & 0.16 & 3' UTR & & \\
\hline $3741 *$ & $\mathrm{G}$ to $\mathrm{C}$ & 0.11 & 8426 & $\mathrm{C}$ to $\mathrm{T}$ & 0.13 \\
\hline $3815^{*}$ & $\mathrm{C}$ to $\mathrm{A}$ & 0.16 & & & \\
\hline
\end{tabular}

*Transversion.

taurus. Among the Indian buffalo breeds 19 SNPs were observed with varying frequencies (Table 3 ). Only 5 changes were transversions and 14 transitions. These SNPs in Indian buffalo breeds are novel and are reported here for the first time. Although these SNPs are not expected to have any functional significance but they may be useful as genetic markers in association studies for milk fat and milk yield.

\section{References}

Annual Report (2006) Department of Animal Husbandary and Dairying, Government of India, New Delhi. www.dahd. nic.in

Cases S, Smith SJ, Zheng YW, Myers HM, Lear SR, Sande E, Novak S, Collins C, Welch CB, Lusis AJ, et al. (1998) Identification of a gene encoding an acyl CoA:diacylglycerol acyltransferase, a key enzyme in triacylglycerol synthesis. Proc Natl Acad Sci USA 95:13018-13023.

Grisart B, Farnir F, Karim L, Cambisano N, Kim J, Kvasz A, Mni M, Simori P, Frere J, Coppieters W, et al. (2004) Genetic and functional confirmation of the causality of the DGAT1 K232A quantitative trait nucleotide in affecting milk yield and composition. Proc Natl Acad Sci USA 101:2308-2403.

Kaupe B, Winter A, Fries R and Erhardt G (2004) DGAT1 polymorphism in Bos Indicus and Bos taurus cattle breeds. J Dairy Res 71:182-187.

Kuhn CH, Thaller G, Winter A, Bininda-Emonds O, Kaupe B, Erhardt G, Bennewitz J, Schwerin M and Fries R (2004) Evidence for multiple alleles at the DGAT1 locus better explains a quantitative trait locus with major effect on milk fat content in cattle. Genetics 167:1873-1881.
Lacorte GA, Machado MA, Martinez ML, Campos AL, Maciel RP, Verneque RS, Teodoro RL, Peixoto MGCD, Carvalho MRS and Fonseca CG (2006) DGAT1 K232A polymorphism in Brazilian cattle breeds. Genet Mol Res 5:475-482.

Rincón G, Armstron E and Postiglioni A (2006) Analysis of the population structure of Uruguayan Creole cattle as inferred from milk major gene polymorphisms. Genet Mol Biol 29:491-495.

Smith SJ, Cases S, Jensen DR, Chen HC, Sande E, Tow B, Sanan DA, Raber J, Eckel RH and Farese Jr RV (2000) Obesity resistance and multiple mechanisms of triglyceride synthesis in mice lacking Dgat. Nat Genet 25:87-90.

Spelman RJ, Ford CA, McElhinney P, Gregory GC and Snell RG (2002) Characterization of the DGAT1 gene in the New Zealand dairy population. J Dairy Sci 85:3514-3517.

Tantia MS, Vijh RK, Mishra BP, Mishra B, Bharani Kumar ST and Sodhi M (2006) DGAT1 and ABCG2 polymorphism in Indian cattle (Bos indicus) and buffalo (Bubalus bubalis) breeds. BMC Vet Res 2:32.

Thaller G, Kuhn C, Winter A, Ewlad G, Bellmann O, Wegner J, Zuhlke H and Fries R (2003) DGAT1, a new positional and functional candidate gene for intramuscular fat deposition in cattle. Anim Genet 34:354-357.

Weller JI, Golik M, Seroussi E, Ezra E and Ron M (2003) Population-wide analysis of a QTL affecting milk-fat production in the Israeli Holstein population. J Dairy Sci 86:2219-2227.

Winter A, Kramer W, Werner F, Kollers S, Kata S, Durstewitz G, Buitkamp J, Womack W, Thaller G and Fries R (2002) Association of a lysine-232/alanine polymorphism in a bovine gene encoding acyl-CoA:diacylglycerol acyltransferase (DGAT1) with variation at a quantitative trait locus for milk fat content. Proc Natl Acad Sci USA 99:9300-9305. Associate Editor: Luiz Lehmann Coutinho 